\title{
Le rôle des exemples en français dans la réflexion linguistique de Ferdinand de Saussure
}

\author{
Marie-José Béguelin \\ Université de Neuchâtel
}

Le système est un procès incessant de corrélation.

(N. La Fauci, Principes de linguistique rationnelle, Pisa, Edizioni ETS, 2005, 24)

\begin{abstract}
Résumé. L'année 2016 marque, on le sait, le centième anniversaire de la parution du Cours de linguistique générale de Ferdinand de Saussure (CLG). Mais le hasard veut qu'elle corresponde aussi au vingtième anniversaire de la découverte, dans l'orangerie de l'hôtel familial à Genève, de documents autographes jusqu'alors inconnus (ÉLG = Saussure 2002, $\mathrm{SL}=$ Saussure 2011). Bien que fragmentaires et d'interprétation délicate, ces manuscrits, notamment ceux connus sous le titre De l'essence double du langage, ont apporté un éclairage nouveau sur la pensée linguistique de Saussure ; ils ont privé de légitimité, sur des points importants, le contenu du CLG édité par Bally et Sechehaye. Dans ce contexte épistémologique peu banal, quels enseignements la linguistique et l'étude descriptive d'une langue comme le français peuvent-elles encore puiser, voire (re)découvrir, dans la pensée de Saussure sur le langage? Tel est le thème que je développerai dans le cadre de cette étude, m'associant ainsi aux bilans entrepris un peu partout en cette période anniversaire. L'objectif sera double. D'une part, il s'agira de rappeler le rôle que jouent les exemples en français dans la réflexion linguistique de Saussure, appréhendée dans les textes autographes ou à travers les notes d'étudiants inspiratrices du CLG - sans omettre les exemples écartés à tort par Bally et Sechehaye. D'autre part, il s'agira de revisiter, à la faveur de cet examen, quelques notions ou dualités d'usage commun, intimement liées au nom de Saussure, mais maniées souvent aujourd'hui de manière réductrice, et sans que l'impact scientifique en ait été pleinement mesuré. Par le prisme des arguments tirés du français, il sera ainsi question du principe de différentialité, de ses incidences sur le calcul du sens, des problèmes associés à la notion d'identité diachronique, du statut de la parole par rapport à la langue, des relations entre linguistique externe et linguistique interne.
\end{abstract}

Abstract. The role of French examples in the linguistic reflections of Ferdinand de Saussure. The year 2016 marks, as we know, the hundredth anniversary of the publication of the Course in General Linguistics by Ferdinand de Saussure (CGL). But it corresponds by coincidence also to the twentieth anniversary of the discovery, in the orangery of the family residence at Geneva, of previously unknown autograph documents (Saussure 2002, Saussure 2011). Although fragmentary and challenging to interpret, these manuscripts, notably those known under the title of On the Dual Essence of Language, have cast a new light on the linguistic thought of de Saussure; they have undercut, on important points, the contents of the CGL edited by Bally and Sechehaye. In this important epistemological context, what can linguistics and the descriptive study of a language such as French still offer, or even (re)discover, in de Saussure's thinking on language? This is the topic that I will develop in this study, taking the opportunity to join others in the reviews undertaken during this 
anniversary period. The objective will be dual. On the one hand, the study examines the role played by the French examples in the linguistic reflections of de Saussure, such as presented in the autograph texts or the notes of students that inspired the CGL — without omitting the examples wrongly discarded by Bally and Sechehaye. On the other hand, it revisits, based on this examination, some notions or dualities of common usage, intimately linked to the name of de Saussure, but often handled nowadays in a reductionist manner, and without their scientific impact being fully appreciated. Through the prism of the arguments drawn from French, I will deal with the principle of differentiation, of their impact on the sense making, of problems associated with the notion of diachronic identity, of the status of parole with regard to langue, the relationship between external linguistics and internal linguistics.

\section{Un double anniversaire}

L'année 2016 marque, on le sait, le centième anniversaire de la parution du Cours de linguistique générale de Ferdinand de Saussure'. Mais le hasard veut qu'elle corresponde aussi au vingtième anniversaire de la découverte, dans l'orangerie de l'hôtel familial à Genève, de documents autographes jusqu'alors inconnus ${ }^{2}$ (ÉLG $=$ Saussure 2002, SL = Saussure 2011). Bien que fragmentaires et d'interprétation délicate, ces manuscrits, notamment ceux qui ont été publiés sous le titre De l'essence double du langage ${ }^{3}$, apportent un éclairage nouveau sur la pensée linguistique de Saussure ; ils privent de légitimité, sur des points importants, le contenu du CLG édité par Bally et Sechehaye ${ }^{4}$.

Dans ce contexte épistémologique peu banal, quels enseignements la linguistique et l'étude descriptive d'une langue comme le français peuvent-elles encore puiser, voire (re)découvrir, dans la pensée de Saussure sur le langage ${ }^{5}$ ? Tel est le thème que je développerai dans la présente contribution.

L'objectif de mon étude est double. Il s'agira d'abord de rappeler le rôle que jouent les exemples en français dans la réflexion linguistique de Saussure, envisagée dans ses textes autographes ou à travers les notes inspiratrices du $\mathrm{CLG}^{6}$. Il s'agira ensuite, à la faveur de cet examen, de faire le point sur quelques notions et dualités d'usage commun, intimement liées au nom de Saussure ${ }^{7}$, mais souvent envisagées aujourd'hui de façon réductrice, sur la base d'informations de seconde main et sans que l'impact scientifique en ait été pleinement

\footnotetext{
${ }^{1}$ Désormais CLG (= Saussure 1916).

${ }^{2}$ Voir le récit de cette découverte dans SL 9-10.

${ }^{3}$ Il s'agit d'un lot de textes à l'état de brouillons, contenus dans une douzaine d'enveloppes sur lesquelles Saussure avait apposé le titre De la double essence du langage (ou en abrégé De l'essence double, De la double essence, etc.) Ils ont été rédigés en vue d'un « opuscule » (selon son propre terme) auquel Saussure a travaillé à partir de l'année 1891.

${ }^{4}$ Loin d'avoir joué le rôle de simples éditeurs, Bally et Sechehaye ont eux-mêmes planifié et rédigé le CLG, à partir de notes d'étudiants prises lors des trois Cours de linguistique générale que Saussure a donnés à Genève entre 1907 et 1911. Ni l'un ni l'autre n'avait assisté en personne à ces enseignements. L'édition synoptique d'Engler (CLG/E) demeure l'instrument de référence qui permet de confronter le CLG à ses sources. Voir aussi désormais Sofia, 2016.

${ }^{5}$ Cette question a inspiré ces dernières années plusieurs monographies et ouvrages collectifs (p. ex. Bouquet 1997, Fehr 2000, Depecker 2009, Bronckart \& al. 2010, Rastier 2015, Coursil 2015 ...) ; anniversaire oblige, elle est au programme de plusieurs colloques internationaux réunis sous le label CLG2016.

${ }^{6} \mathrm{Cf}$. CLG/E ; Cours III C ; Leçons

${ }^{7}$ Dans l'introduction de son édition critique du CLG, De Mauro a dressé une liste des nombreux mots clés de la linguistique qui apparaissent pour la première fois dans le CLG ou qui y reçoivent, dans ses termes, «une sanction définitive dans une acception déterminée »(CLG/DM p. IV).
} 
mesuré ${ }^{8}$. Par le prisme des arguments tirés du français, il sera ainsi question du principe de différentialité, de ses incidences sur le calcul du sens et sur l'approche de la diachronie, du statut de l'opposition langue / parole, du rôle attribué à la conscience du locuteur, des relations entre linguistique externe et linguistique interne.

\section{L'œuvre de Saussure et sa transmission}

Avant d'entrer dans le vif du sujet, je rappellerai brièvement l'itinéraire intellectuel de Saussure (1857-1913) et les voies complexes par lesquelles son œuvre linguistique nous est parvenue, suscitant une abondante littérature à vocation exégétique et philologique.

Ferdinand de Saussure - présenté parfois comme un philosophe du langage — n'était autre, aux yeux de ses contemporains, qu'un professeur de grammaire comparée des langues indoeuropéennes estimé de ses pairs et dont l'enseignement captivait ses étudiants ${ }^{9}$. Les cours qu'il dispense, à Paris pendant une dizaine d'années (EPHE), puis à Genève à partir de 1891, portent essentiellement sur les langues anciennes (langues germaniques : gotique, vieux hautallemand; latin, grec, sanskrit, lituanien), dans une perspective comparative et reconstructive $^{10}$. Ces langues, Saussure n'en avait pas seulement une connaissance métalinguistique, acquise dans les grammaires, mais il en maîtrisait plusieurs de façon intime, via une fréquentation assidue des textes ${ }^{11}$. Les publications réalisées du vivant de Saussure $\left(\right.$ Recueil $\left.^{12}\right)$ sont relativement peu nombreuses : elles relèvent presque toutes de cette sphère d'études qu'il a, dans son Mémoire, éclairée de ses découvertes lumineuses (cf. notes 12 et 27). Les trois Cours de linguistique générale donnés à Genève en 1907, 1908-1909 et 19101911, et qui sont à la source du CLG posthume, demeurent imprégnées de cette expérience approfondie de comparatiste.

Cependant, dès les années 1880, Saussure développe une réflexion générale sur le langage ${ }^{13}$. Il s'interroge sur la détermination des unités linguistiques (qui, au contraire des objets physiques étudiés par les sciences naturelles, ne sont pas des «choses » tombant sous le sens) ; sur leur mode d'existence et leur statut réciproque ; sur la raison d'être des analyses du savant et leur légitimité. De fil en aiguille, sa réflexion s'étend aux propriétés du signe, au

\footnotetext{
${ }^{8}$ Quand il n'est pas abordé par le prisme d'ouvrages de vulgarisation plus ou moins bien informés, le CLG fait souvent l'objet d'une lecture sélective, ne retenant que les principes généraux placés en tête de volume par les éditeurs (cf. Redard 1978; CLG DM p. XV). Les développements relatifs à la linguistique diachronique, à la géographie linguistique, demeurent méconnus.

${ }^{9}$ CLG/DM 319-394 et Joseph 2012 présentent des renseignements détaillés sur la carrière de Saussure. Voir récemment, sur l'apport de Saussure à la connaissance des langues classiques, Forel \& al. 2015.

${ }^{10}$ Il a cependant donné aussi, de 1900 à 1909, un cours annuel de «Versification française » dont les notes préparatoires sont conservées : voir à ce sujet Arrivé 2009.

${ }_{11}^{11}$ En témoignent notamment ses recherches sur la poétique et les anagrammes (cf. infra note 24).

${ }^{12}$ Ce Recueil des publications scientifiques de 640 pages reproduit le Mémoire sur le système des voyelles dans les langues indo-européennes de 1879 [1878], paru lorsque Saussure avait 21 ans, et qui demeure le principal ouvrage publié par ses soins. Le Mémoire condense une série de découvertes et d'intuitions (structure des racines et des cellules morphologiques, "coefficients sonantiques ») qui ont profondément marqué le devenir de la grammaire comparée (cf. Kuryłowicz 1978, Vallini 2013, Béguelin 2000, Utaker, 2002). Le Mémoire a valu à Saussure une célébrité précoce dans le milieu des comparatistes, mais aussi des attaques et des désillusions. Le Recueil inclut en outre la thèse de doctorat sur le génitif absolu en sanskrit, d'ambition plus modeste, ainsi que diverses études à caractère étymologique.

${ }^{13} \mathrm{Ne}$ serait-ce que pour défendre, contre ses détracteurs, les positions adoptées dans son Mémoire (cf. note 12)
} 
caractère à la fois individuel et social de la langue, aux différents points de vue (historique ou «synoptique », externe ou interne) sous lesquels il est possible de l'appréhender, aux modalités de sa transmission.

Aux yeux de Saussure, l'entité linguistique par excellence est, on le sait, le signe, conçu comme l'union indissociable d'une forme vocale et d'un concept (un signifiant et un signifié), qui sont psychiques l'un comme l'autre et constituent les deux faces d'une même réalité ${ }^{14}$. Défini de manière exclusivement différentielle, le signe est dénué de substrat positif : "tout signe repose purement sur un co-status négatif » (ÉLG 230, note Status et motus). Nous reviendrons aux $\S \S 3.3-3.4$ sur les conséquences de cet axiome central de la théorie. Dans sa vaste entreprise d'ordonnancement, Saussure distingue de surcroît la langue (comme système de signes) et la parole («force active et origine véritable des phénomènes qui s'aperçoivent ensuite peu à peu dans l'autre partie du langage $\left[i\right.$. e. la langue, MJB] ${ }^{15}$; l'état et l'événement, reflets de deux points de vue nécessaires, mais inconciliables ${ }^{16}$ sur la langue, le point de vue synchronique et le point de vue diachronique ${ }^{17}$; le syntagme ou "parole effective », lieu des relations combinatoires, et la parallélie ou «parole potentielle », lieu des relations associatives entre unités ( $\mathrm{SL} \mathrm{n}^{\circ} 83=$ ÉLG 61, Essence double).

Issu d'une famille de savants et de naturalistes "rompus aux techniques de la taxinomie et aux raisonnements de la systématique »(Laks 2012: 96), Saussure aspire à faire de la linguistique une discipline dont l'objet - quoique particulièrement difficile à saisir — soit circonscrit avec autant de rigueur que celui de la physique, de la chimie ou de l'astronomie. Les approches étymologistes en faveur à son époque ne peuvent le satisfaire : car elles manipulent sans méthode, en mélangeant époques et points de vue, des entités au statut ontologique hasardeux, coupées de leur environnement paradigmatique et de leur contexte d'utilisation. Or pour Saussure, inspiré par ses recherches sur le système morphophonologique de l'indo-européen, un fait linguistique envisagé tout seul n'a, littéralement, pas d'existence :

\footnotetext{
${ }^{14} \mathrm{Cf}$ : : « Il est aussi littéralement vrai de dire que le mot est le signe de l'idée que de dire que l'idée est le signe du mot » (SL n ${ }^{\circ} 47$ = ÉLG 44, Essence double); ou encore : " Il est donc entièrement illusoire d'opposer à un aucun instant le signe à la signification. Ce sont deux formes du même concept de l'esprit, vu que la signification n'existerait pas sans un signe, et qu'elle n'est que l'expérience à rebours du signe, comme on ne peut pas découper une feuille de papier sans entamer l'envers et l'endroit de ce papier, du même coup de ciseaux. » (ÉLG 96, Nouveaux Item). Dans ces deux extraits, il faut entendre signe au sens de signifiant.

${ }^{15}$ ÉLG 273, Rapport sur la création d'une chaire de stylistique. La présentation de la distinction langue-parole a été altérée dans le CLG, engendrant des contresens durables sur la position de Saussure à cet égard. Cf. $\S 3.4$ et notes 17 et 42 .

${ }^{16}$ Cf. p. ex. : « Partout l'état historique et l'état conscient sont deux états qui s'opposent. Ce sont les deux voies du signe. D’où la difficulté, mais la nécessité, de ne les mélanger nulle part en rien. » (ÉLG 117, Anciens Item).

${ }^{17}$ Alors que le couple langue-parole est chez Saussure (à l'instar du signe) une dualité constituant le champ de la linguistique proprement dite (notes 15 et 16), synchronie et diachronie représentent pour lui une dichotomie et relèvent, au moins tendanciellement, de deux sciences différentes : "Comme quoi tout (?) fait de langue demande séparation entre les points de vue diachronique et synoptique. » ( $\mathrm{SL} \mathrm{n}^{\circ} 42$ = ÉLG 66, Essence double); « car on peut se demander même si cette distinction, une fois bien reconnue et comprise, permet encore l'unité de la linguistique, n'a pas pour conséquence de nous faire voir dans la langue deux objets entièrement différents, nécessitant deux sciences que je ne qualifierais pas même de parallèles, mais de [ ] » (texte inachevé, ÉLG 233, Status et motus. Notes pour un livre de linguistique générale, 2).
} 
Tout fait linguistique consiste en un rapport, et consiste en rien d'autre qu'en un rapport. (ÉLG 263, Note Sémiologie)

Le premier caractère universel du langage est de vivre au moyen de différences et de différences seules, sans aucune mitigation comme celle qui proviendrait de l'introduction d'un terme positif quelconque à un moment quelconque. (ÉLG 264, ibid.)

D'où l'inanité qu'il y a à prétendre étudier une forme quelle qu'elle soit en abstraction des formes ambiantes dont elle retire sa valeur, i. e. son existence en tant que fait linguistique.

S'agissant de cette chose immatérielle qu'est la langue, où convient-il de situer l'objet d'étude? Et sur quel guide s'appuyer pour éviter de «tirer des conclusions absurdes sur le Langage de l'œuvre de la grammaire ${ }^{18}$ ? Cet objet et ce guide, Saussure les situe d'emblée dans le «sentiment de la langue chez ceux qui la parlent»" autrement dit dans la « conscience du sujet parlant », qui fournit le seul accès possible aux structures grammaticales concrètes d'un état de langue donné. L'étymologie, pur fait du savant, est impitoyablement exclue de la linguistique ${ }^{20}$; Saussure lui oppose la science du langage véritable, qu'il baptise du nom de sémiologie ou de signologie (ÉLG 262-266) ${ }^{21}$, et dont la tâche est d'envisager la 'langue' « comme langue et non pas comme résultat d'états précédents » (SL n $100=$ ÉLG 70, Essence double). Face à la perspective historique toute-puissante au $\mathrm{XIX}^{\mathrm{e}}$ siècle, Saussure réintroduit ainsi, dans l'étude du langage humain, le point de vue du sujet confronté à la pure contemporanéité des signes dont il se sert.

À plus d'un siècle de distance, la doctrine sommairement résumée ci-dessus n'a perdu ni son impact, ni son pouvoir de questionnement (cf. Bronckart \& al. 2010 ; La Fauci 2011 ; Rastier 2015). Sous la forme tempérée et parfois gauchie qu'en fournit le CLG, elle a valu à Saussure le titre posthume d'inventeur de la sémiologie et de père de la linguistique moderne; de son vivant cependant, Saussure n'a jamais donné de forme définitive à ses réflexions ${ }^{22}$, dont les échos nous sont parvenus par le truchement de ses étudiants ou grâce à des fragments autographes d'un intérêt inappréciable, mais à l'état d'ébauches (ÉLG, SL). Quant au CLG, rédigé par Bally et Sechehaye sur la base de notes d'étudiants (cf. Leçons), il fournit de la

\footnotetext{
${ }^{18}$ ÉLG 204, Notes pour un article sur Whitney.

${ }^{19}$ L'expression est tirée d'une note de 1884, publiée in Marchese $2015: 185$. Quelques lignes plus loin apparait la formule « conscience de ceux qui la parlent».

${ }^{20}$ Les notes de Riedlinger relèvent ainsi : «L'étymologie n'a aucune place dans la linguistique ». (CLG/E 2364, I R); ou encore : «nous ne parlons pas évolutivement par étymologie, mais par valeurs existantes » (CLG/E 1601 , II R).

${ }^{21}$ ÉLG 262-266, Note Sémiologie. La sémiologie englobe la morphologie : «Le vrai nom de morphologie serait : la théorie des signes et non des formes. » (ÉLG 182, Note Morphologie).

${ }^{22}$ En mai 1907, dans un courrier relatif à un article sur l'astronomie chinoise publié par son frère Léopold, Saussure livre une confidence indirecte, mais révélatrice : «Je crains une seule chose, c'est que Léopold ne s'enfonce jusqu'aux derniers principes - comme il m'est arrivé cent fois -, et ne projette alors un gros ouvrage qui ne paraîtra jamais, au lieu d'une suite d'articles. » (Lettre de Saussure à son ami Jean-Elie David, 18.5.1907, in Meijía Quijano $2014: 467$, mes caractères gras). Benveniste a vu on le sait dans le silence éditorial où s'est installé Saussure le signe d'un «drame de la pensée »: "Saussure s'éloignait de son époque dans la mesure où il se rendait peu à peu maître de sa propre vérité, car cette vérité lui faisait rejeter tout ce qui était enseigné alors au sujet du langage. Mais, en même temps qu'il hésitait devant cette révision radicale qu'il sentait nécessaire, il ne pouvait se résoudre à publier la moindre note qu'il n'eût d'abord assuré les fondements de la théorie. » (Benveniste, $1966=1963: 37$; voir aussi ibid. le contenu souvent cité de la lettre à Meillet de janvier 1894, reproduit également in CLG/DM 355).
} 
pensée de Saussure un témoignage doublement indirect, dont la confrontation avec ses sources révèle des distorsions, des omissions, voire des contresens ${ }^{23}$. Faute des compétences requises pour comprendre les textes du Recueil, le lecteur aborde donc aujourd'hui, par la force de choses, l'œuvre de Saussure au travers de documents qui ne sont pas de sa main (CLG, Leçons), ou qu'il n'avait pas choisi de divulguer (ÉLG, SL, Phonétique, Théorie des sonantes, Saussure $2013 \ldots{ }^{24}$ ).

\section{Place des langues romanes et du français}

\subsection{Les langues vivantes comme moyen de contrôle}

Saussure est parfois présenté, on l'a dit plus haut, comme un théoricien, un philosophe, voire un métaphysicien; mais on peut voir en lui, de manière tout aussi légitime, un empiriste adepte de la méthode inductive. Comme il s'en explique dans sa Première conférence à l'Université de Genève (1891), l'observation approfondie des manifestations linguistiques et la quête de principes généraux sont indispensables l'une comme l'autre à la science du langage ${ }^{25}$. De surcroît, «l'extrême spécialisation peut seule servir efficacement l'extrême généralisation », et la connaissance approfondie d'une langue ou d'une famille de langues est plus profitable à la science qu'un polyglottisme de façade ${ }^{26}$. Ainsi Saussure rend-il hommage aux études germaniques et slaves, de même qu'au travail précurseur des romanistes :

On a parlé un peu prématurément d'une science du langage. C'était à une époque où personne encore, à part de rares romanistes, ne pouvait avoir conçu l'idée de ce qu'est LA LANGUE, ni même UNE langue dans son évolution. (ÉLG 265, note Sémiologie, mes caractères gras)

Saussure est convaincu d'autre part, à l'instar de son maître et collègue Bréal et du sanskritiste américain Whitney, de «l'importance des vérifications modernes de chaque phénomène » (ÉLG 234, Anciens documents) : dès son Mémoire, l'observation des langues vivantes (notamment celle des phénomènes d'alternance, cf. note 34) lui sert de clé de

\footnotetext{
${ }^{23}$ Cf. note 4 supra. Ce texte établi hors de toute déontologie philologique (cf. Kyheng 2007), cette vulgate qualifiée d'apocryphe par des commentateurs avertis et dont les défauts ont été relevés dès sa parution (cf. Bouquet 2010), a néanmoins fait la notoriété de Saussure. Dans le même temps, on cessait peu à peu de lire son chef-d'œuvre autographe, le Mémoire sur le système des voyelles dans les langues indo-européennes de 1879 (note 12) - qui contient une mise en œuvre précoce de la notion de la langue (Béguelin 2011a).

${ }^{24}$ Pour mémoire, rappelons que Saussure a beaucoup travaillé sur la mythologie germanique et, dans un relatif secret, sur les principes de versification et la présence d'anagrammes dans le texte poétique. Ses recherches à ce sujet ont été elles aussi diffusées à titre posthume (Starobinski 1971, Testenoire 2013, Saussure 2013; Légendes).

${ }^{25}$ « Vouloir étudier le langage sans se donner la peine d'en étudier les diverses manifestations qu'évidemment sont les langues est une entreprise absolument vaine, et chimérique ; d'un autre côté vouloir étudier les langues en oubliant que ces langues sont primordialement régies par certains principes qui sont résumés dans 1'idée de langage est un travail encore plus dénué de toute signification sérieuse, de toute base scientifique véritable. » (ÉLG 146, Première conférence à l'Université de Genève, novembre 1891).

${ }_{26}$ «Ce ne sont pas des linguistes comme Friedrich Müller, de l'Université de Vienne, qui embrassent à peu près tous les idiomes du globe, qui ont jamais fait faire un pas à la connaissance du langage ; mais les noms qu'on aurait à citer dans ce sens seraient des noms de romanistes comme M. Gaston Paris, M. Paul Meyer, M. Schuchardt, des noms de germanistes comme M. Hermann Paul, des noms de l'école russe s'occupant spécialement du russe et du slave, comme M. N. Baudouin de Courtenay, M. Kruszewski. » (ÉLG 147, Première conférence à l'Université de Genève, novembre 1891).
} 
décryptage pour aborder les états de langue révolus ${ }^{27}$. En témoigne ce passage de la Première conférence à l'Université de Genève où le Professeur dénonce, Gaston Paris à l'appui, les représentations erronées induites par les noms attribués aux états de langue successifs, par exemple dans la phrase triviale le français vient $d u$ latin :

Il suffit d'y réfléchir un instant, puisque tout est contenu dans cette simple observation : chaque individu emploie le lendemain le même idiome qu'il parlait la veille et cela s'est toujours vu. Il n'y a donc eu aucun jour où on ait pu dresser l'acte de décès de la langue latine, et il n'y a eu également aucun jour où on ait pu enregistrer la naissance de la langue française. Il n'est jamais arrivé que les gens de France se soient réveillés en se disant bonjour en français, après s'être dit bonne nuit en latin. (ÉLG 152, Première conférence à l'Université de Genève, novembre 1891) ${ }^{28}$.

Alors que les désignations latin et français laissent inférer deux langues, deux "choses » distinctes, l'expérience quotidienne, signale Saussure, fait "crouler ce dualisme de carton » (ÉLG 166) ; en linguistique, une simple introspection, à la portée du locuteur de bon sens, peut donc prêter à généralisation ${ }^{29}$.

Dans ses notes préparatoires comme dans son enseignement, Saussure exploite abondamment le français ${ }^{30}$, langue qu'il partage avec ses auditeurs ${ }^{31}$. Pour illustrer l'universalité d'un

\footnotetext{
${ }^{27}$ Cf. : «Les deux choses, une bonne généralisation sur le langage, qui peut intéresser qui que ce soit, ou une saine méthode à proposer à la grammaire comparée pour les opérations précises de chaque [ ] sont en réalité la même chose. » (ÉLG 204-205, Note pour un article sur Whitney). Saussure écrit, en 1885 déjà, à propos des lois phonétiques : «Le meilleur moyen de se rendre compte de la nature des 'lois phonétiques' est d'observer ce qui se passe en présence d'une prononciation vicieuse, c'est-à-dire individuelle ou dialectale, soit chez la personne qui prononce mal, soit chez celui qui l'écoute. » (Note datée de mars 1885, in Marchese 2015: 186; des illustrations sont données ensuite). L'auteur du Mémoire est, il faut le rappeler, le premier à avoir envisagé l'indo-européen comme une langue parcourue de régularités grammaticales et fonctionnelles, ce qui a permis la fameuse découverte des coefficients sonantiques (connus aujourd'hui sous le nom de laryngales indoeuropéennes). Sur la méthode comparative de Saussure, cf. p ex. Watkins, 1978, Vallini 2013, Utaker 2002, Bergounioux 2010, Béguelin 2003 et 2011a.

${ }^{28}$ L'absence de rupture dans la transmission linguistique a son pendant au plan géographique, et à moins de circonstances particulières, les divers noms donnés aux dialectes de même souche ne correspondent à aucune scission réelle dans la continuité des parlers : «Exemple dans un cas qui est tout près de nous : si l'on marche en diagonale à travers la France du Pas de Calais aux Alpes, puis en continuant jusqu'à Turin, il n'y a pas un endroit précis où l'on puisse dire : je quitte le français et j'entre dans l'italien. » (ÉLG 316, Notes pour le Cours III).

${ }^{29}$ Une quinzaine d'années plus tard, dans ses Cours de linguistique générale, Saussure poursuit ses attaques contre les pièges ou «cavernes » de la linguistique. Le cas de l'orthographe française est utilisé parmi d'autres pour montrer la nécessité de ne pas rester « servilement attaché à la lettre » (CLG/E 94, III C 5) - même quand on ne dispose que de textes pour documenter un état de langue révolu. Saussure exemplifie abondamment les vicissitudes de la relation graphie-phonie, notamment par le cas de la séquence graphique oi (cf. roi, loi, croix), restée stable du XIII ${ }^{\mathrm{e}}$ siècle à nos jours alors que la prononciation correspondante passait successivement de oi à $o e ̀, o a$, et $w a(\mathrm{CLG} / \mathrm{E} 523)$ : «l'écriture met une unité factice à ce qui est échelonné dans le temps et l'espace » (CLG/E 479, II R). Au chapitre des facteurs qui influencent le devenir d'une langue, Saussure signale aussi l'intervention d'organismes tels que l'Académie française, et le « fait non linguistique d'une immense littérature qui impose une certaine langue de l'extérieur» (Leçons, I R 6) - autant de phénomènes externes qui ne demeurent pas sans effet sur l'état du système. Voir à ce sujet les propos de Regard qui a «plus d'une fois entendu F. d. S. expliquer par des conditions extérieures non seulement les changements linguistiques, mais la conservation de certains traits » (cité in CLG/DM 347).

${ }^{30}$ Saussure recourt aussi à d'autres langues telles que l'allemand, l'anglais, l'italien, le tchèque, l'hébreu, le chinois ou les parlers gallo-romans; son argumentation repose en outre sur une série de comparaisons dont certaines sont célèbres : partition musicale, jeu d'échecs, peinture et couleurs, chimie, économie, physiologie, numismatique, etc.
} 
phénomène, quel moyen plus efficace que d'éclairer les exemples historiques à l'aide de données contemporaines? Ainsi, dans ce passage consacré à l'analogie :

Contemporain : par exemple j'achète, nous achetons. Une femme ne dit plus je me décollette mais je me décolte. Magnifique cas d'analogie! Il est clair, non pas qu'il faut dire (car il ne faut rien dire : car tout ce qu'on dit a sa raison d'être) - mais il est clair qu'on a dit jusqu'à une époque récente [je me décollette, nous nous décolletons] comme j'achète, [nous achetons]. Très possible qu'on dise une fois j'achte (j'ai déjà entendu je cachte). » (ÉLG 162, Deuxième conférence à l'Université de Genève, novembre 1891 ; cf. aussi le passage cité note 35)

Ces quelques lignes sont révélatrices. Outre l'intérêt manifesté pour la langue orale non académique (cf. De Palo 2016: 50), on y lit l'enthousiasme du savant face à un beau spécimen «Magnifique cas d'analogie ! , le dédain pour la norme qui signale une approche véritablement scientifique des faits (« car il ne faut rien dire : car tout ce qu'on dit a sa raison d'être »), enfin une ambition intellectuelle qui vise non seulement à décrire, mais aussi à prédire («Très possible qu'on dise une fois $j$ 'achte »)...

Les exemples empruntés au français sont légion chez Saussure et ils apparaissent souvent à l'occasion de raisonnements décisifs. Il y a là tout un chantier dont il ne me sera possible de donner, dans ces lignes, qu'un modeste aperçu. Les spécimens qui seront présentés ci-après ont été choisis pour leur caractère emblématique et pour leur pertinence dans des débats d'actualité ; ils auront trait successivement à la définition des "réalités » linguistiques (3.2), au principe de différentialité et à ses domaines d'application (3.3), aux défis posés par l'étude scientifique de la diachronie (3.4) enfin au statut accordé à la conscience du locuteur (3.5). Si ces exemples peuvent sembler hétéroclites à première vue, ils visent tous à étayer, on le verra, un petit nombre de grands principes en faveur desquels Saussure plaide avec une remarquable constance.

\subsection{Unités et réalités linguistiques}

La première tâche qui se présente au linguiste est de s'assurer que les entités qu'il postule ont une existence dans la réalité, autrement dit qu'elles méritent le nom d'entités linguistiques (cf. $\S 2$ ) ; le problème se pose de manière aiguë en diachronie (voir infra § 3.4), mais aussi, comme le signale ce passage des notes de Riedlinger, dans la temporalité du discours :

La question des identités peut se confondre en partie avec celle des unités. Elle n'est qu'une complication - du reste féconde - de cette question. Sur quoi faisons-nous reposer l'identité de calidus et de chaud ( $\check{s}$ ) ou bien de despectus avec dépit ? La chaîne phonique dans ces deux derniers mots est différente, la signification est loin d'être identique. En quoi consiste-telle, cette identité ? Mais ne nous imaginons pas que là soit la grande question : il est tout

\footnotetext{
${ }^{31}$ Dans les ÉLG, le lemme français, tous sens confondus, apparaît à 87 reprises, contre 104 occurrences de latin, 51 de grec et 33 d'allemand. Dans les Leçons, avec123 occurrences, français devance latin (114 occurrences), lui-même suivi d'allemand (100 occurrences) puis de grec (91 occurrences). Ces comptages indicatifs ont été réalisés à partir des documents préparatoires des ÉLG et des Leçons; je remercie Simon Bouquet de les avoir aimablement mis à ma disposition. La présence du français est moindre, comme on peut s'y attendre, dans les publications réunies dans le Recueil; les éditeurs du volume, Bally et Gautier, ne retiennent, sous la rubrique Français de l'index, que cinq noms propres de lieux dont Saussure a exposé l'histoire dans le cadre de sociétés savantes.
} 
aussi intéressant de se demander sur quoi nous faisons reposer l'affirmation de l'identité de «Messieurs!» et «Messieurs!». Assurément, il y a là deux actes successifs! Il faut se référer à un lien quelconque. Quel est-il ? (CLG/E 1771, 1759-1761, 1765, II R)

La quête du lien qui fait l'unité linguistique prend un tour crucial dans l'étude des faits d'homonymie, dont le français abonde. Saussure relève ainsi dans l'Essence double qu'une séquence de sons telle que $m+e+r$ n'a pas d'existence, et n'est pas même attribuable à une langue quelconque, si l'on ne considère l'idée qui s'y rattache ${ }^{32}$. L'entité linguistique, en conclut-il, est duelle, et « lui contester cette dualité ou l'oublier revient directement à lui ôter son existence linguistique, en la rejetant par exemple dans le domaine des faits physiques » (SL n ${ }^{0} 36=$ ÉLG 20). Le commentaire d'un autre exemple, celui du couple cher (adjectif) et Cher (nom du département), fait intervenir de manière décisive le sentiment du locuteur :

On appelle forme une figure vocale qui est déterminée pour la conscience des sujets parlants. $[\ldots]$

Par quoi cette figure vocale est-elle déterminée pour la conscience des sujets parlants?

$1^{\circ}$ Est-ce $<$ comme on pourrait $>$ l'imaginer au premier abord [par les successions identiques de sons identiques] par les sons qui s'y trouvent? - Nullement. Un homme habitant le Cher peut passer sa vie sans se rendre compte que ce nom de son département ne diffère pas, en ses sons, du mot qu'il prononce dans cher ami. (SL n $68=$ ÉLG 49)

La différence de valeur (ou de sphère d'emploi) entre les homonymes cher et Cher peut aller jusqu'à inhiber, chez le sujet parlant, la conscience de leur identité sonore : à l'endroit des historiens absorbés par les vicissitudes de la forme vocale, Saussure en déduit la suprématie de la fonction significative, opérationnelle pour le locuteur ${ }^{33}$.

$\mathrm{Au}$ niveau des sous-unités du mot, ce sont les néologismes, créés par analogie avec des formes contemporaines, qui apportent la preuve du fait qu'une entité est « ressentie » par le sujet parlant, i. e. qu'elle est dotée d'une «existence linguistique »" ${ }^{34}$. Dans la note Morphologie, à côté d'exemples tirés de la flexion nominale athématique du grec, le

\footnotetext{
${ }^{32}$ Voir SL n ${ }^{\circ} 36$ = ÉLG 19-20. Saussure a sans doute en tête l'homophonie qui caractérise la série mer-mèremaire.

${ }^{33}$ Cf. ce passage du Cours II, cité dans la version Riedlinger : « Ce qui est dans le sentiment des sujets parlants, ce qui est ressenti à un degré quelconque, c'est la signification, et on pourra dire alors que le concret réel, pas du tout si facile à saisir dans la langue = ce qui est ressenti, ce qui à son tour = ce qui est significatif à un degré quelconque. Ce qui est significatif se traduit par une délimitation d'unité, c'est la signification qui la crée, elle n'existe pas avant : ce ne sont pas les unités qui sont là pour recevoir une signification. » (CLG/E 1737, II $\mathrm{R}$, mes caractères gras ; voir un peu plus haut la discussion sur moi et mois : CLG/E 1730 II R).

${ }^{34} \mathrm{Cf}$. aussi la productivité de l'alternance (ou apophonie), qui est « le plus important, le plus ordinaire et le plus vaste des effets grammaticaux que peuvent avoir les changements phonétiques » (CLG/E 2403, I R) : «En français, nous avons des séries comme :
}

$$
\begin{aligned}
& \text { pouvons : peuvent } \\
& \text { voulons : veulent } \\
& \text { nouveau : neuve, neuf } \\
& \text { douloureux : douleur }
\end{aligned}
$$

Nous dégageons l'élément de différence et sa variation régulière plus ou moins facilement, avec plus ou moins de conscience, mais enfin, nous le dégageons ; à preuve le mot Babouvisme créé de Babeuf !» (CLG/E 2404, I R ; Babeuf est le nom d'un révolutionnaire français). 
processus de création de nouveaux noms d'agent français en -eur est appelé à la rescousse et modélisé via la célèbre formule de la quatrième proportionnelle :

En français nous avons conscience par exemple d'un élément -eur qui employé d'une certaine façon servira à donner l'idée de l'auteur d'une action : graveur, penseur, porteur.

Question: Qu'est-ce qui prouve que cet élément -eur est réellement isolé par une analyse de la langue?

Réponse: Comme dans tous les cas pareils ce sont les néologismes, c'est-à-dire les formes où l'activité de la langue et sa manière de procéder trouvent à se manifester dans un document irrécusable : men-eur, os-eur, recommenc-eur.

$[\ldots]$

Conclusion : l'analyse morphologique du grammairien, dans la mesure où elle se trouve d'accord avec l'analyse de la langue attestée par les néologismes ou formations d'analogie, ne saurait passer pour un produit de l'abstraction. (ÉLG 184, note Morphologie, mes caractères gras)

Nous ne nous disons pas: je joins l'élément os- et l'élément -eur. Non. Nous procédons toujours par proportion : je grave, ou graver: graveur $=j^{\prime}$ ose ou oser : $x ; x=$ oseur. C'est donc toujours le mot fait qui est notre unité fondamentale. Mais cela n'empêche pas que nous n'accomplissions inconsciemment sur le mot fait la même analyse que le linguiste. Nous dégageons un son relatif à telle ou telle idée particulière, comme oser, penser, et un autre son appelé à marquer une relation déterminée du mot avec cette idée. - Là est la justification de l'analyse morphologique. (ÉLG 193, note Morphologie, mes caractères gras ${ }^{35}$ )

L'usage du nous, très présent dans le second extrait, retient l'attention. La quatrième personne englobe le professeur, sujet de l'énonciation, et ses allocutaires étudiants, considérés en l'occurrence comme de simples locuteurs, exerçant leur langue de manière naïve ; à ce nous inclusif, Saussure confronte un linguiste-type au statut de délocuté, extérieur à la situation de parole. Alors que dans la citation précédente, le grammairien est invité à calquer ses analyses sur celles du sujet parlant, c'est ici, de manière symétrique, au sujet parlant qu'est assignée une activité inconsciente de linguiste ${ }^{36}$. La fusion des points de vue du linguiste et du sujet parlant est au centre de la théorie et de la méthode saussuriennes (cf. $§ 2$ et note 19) : l'appel à l'expérience linguistique commune fournit dès lors un argument privilégié, accréditant par excellence une conception des « réalités » ancrée dans l'activité cognitive et langagière du locuteur.

\footnotetext{
${ }^{35}$ Voir aussi dans les Leçons l'extrait du Cours II consacré aux dérivés de type missionnaire, pensionnaire, etc. : «En allemand, où il est permis de tirer de tout nom un diminutif comme Fräulein de Frau, un auteur pourrait imaginer le premier un diminutif Elephantlein ; de même en français, où l'on forme pensionnaire de pension, réactionnaire de réaction, missionnaire de mission etc., si quelqu'un disait le premier, par exemple pour désigner un parti, pour la ... [répression, etc.], les interventionnaires, les répressionnaires, etc., nous pouvons tout d'abord nous demander si ce processus est le même que celui qui précédemment engendrait honor; ce qui est évident :

$$
\begin{gathered}
\text { mission : missionnaire }=\text { répression }: x \\
x=\text { répressionnaire. } \gg(\mathrm{CLG} / \mathrm{E} 2501-2503, \mathrm{II} \mathrm{R})
\end{gathered}
$$

${ }^{36}$ La création analogique est plus d'une fois illustrée par la flexion verbale dans le langage enfantin : p. ex. cas de venirai dans ÉLG 160, Deuxième conférence à l'Université de Genève, 1891), viendre, venira, traisait (de traire) (CLG/E 2464, 2565-2567, I R, 2591, II R). Concernant le rapport entre " conscience du locuteur » et savoir du linguiste, voir Béguelin $1990 \mathrm{~b}$.
} 


\subsection{La codétermination des entités linguistiques}

Pour Saussure on l'a vu, les entités linguistiques se déterminent entre elles; chacune tire son identité et sa valeur de son opposition avec les autres. Les conséquences sur la méthode du linguiste sont majeures, car " le fait dont il s'occupe n'existe littéralement pas ailleurs que dans la présence de faits opposables » (SL n ${ }^{0} 51=$ ÉLG 65, Essence double). Ce principe de négativité et sa contrepartie, la théorie de la valeur, trouvent notamment confirmation dans les latitudes de prononciation du $r$ français :

En français on peut prononcer sous le son de $r$ deux ou trois consonnes complètement différentes d'articulation et de plus tellement différentes pour l'oreille qu'il n'y a rien qu'on remarque plus immédiatement dans le parler d'un individu. Cependant tous ces sons très différents sont acceptés - pour ainsi dire légalement - comme valant la même chose : [...] (SL $\mathrm{n}^{\circ} 100=$ ÉLG 71-72, Essence double)

Nous tirons de là, d'une manière générale, que la langue repose sur un certain nombre de différences ou d'oppositions qu'elle reconnaît et ne se préoccupe pas essentiellement de la valeur absolue de chacun des termes opposés, qui pourra considérablement varier sans que l'état de langue soit brisé.

La latitude qui existe au sein d'une valeur reconnue peut être dénommée 'fluctuation'. Dans tout état de langue on rencontre des fluctuations. (SL n ${ }^{\circ}$ 63= ÉLG 36, Essence double, mes caractères gras)

La variation ou 'fluctuation' phonétique est, écrit Saussure, «tout à fait caractéristique du principe négatif qui est au fond du mécanisme de la langue » (SL n ${ }^{\circ} 100=$ ÉLG 71, Essence double); il en découle qu'il est vain de rechercher une "valeur absolue » dans les termes d'une opposition linguistique. Pionnière au début des années 1890, cette appréhension des sons en tant que valeurs pures (Coursil 2015), ou éléments « co-définis uniquement par leurs relations taxinomiques » (Laks 2012: 101), a comme on sait irrigué la phonologie jusque dans ses courants les plus actuels (Bergounioux \& Laks, 2013).

Cependant, Saussure fait une application générale et non sectorielle du principe de négativité, qui chez lui n'est pas cantonnée aux "plus élémentaires substructions du langage » (SL $\mathrm{n}^{\circ}$ 100 = ÉLG 71). Ainsi la fluctuation phonétique trouve-t-elle, au plan du signifié, une contrepartie dans la flexibilité de la signification ${ }^{37}$. Cet aspect est discuté dans quelques fragments de l'Essence double d'un intérêt majeur, où Saussure analyse, dans une perspective à la fois intrasystémique et contrastive, les acceptions de termes français tels que soleil, lune, ombre, esprit, chêne, supplice etc. (ÉLG 72-81) ${ }^{38}$. Ces textes proposent une théorie sui generis de la polysémie, en rupture radicale avec les approches lexicographiques fondées sur la notion de trope (cf. De Palo 2016).

Saussure relève d'abord que le signifié d'un mot est, dans un état de langue donné, inépuisable, et qu'il s'applique bien au-delà des sens effectivement constatés ; les bornes

\footnotetext{
${ }^{37}$ Cf. la terminologie testée dans les Anciens Item, où l'« idée invariable et influctuable » est concédée aux noms propres et aux noms géographiques (ÉLG 106, Anciens Item, mes caractères gras).

${ }^{38}$ Il traite aussi par ailleurs, dans cette perspective, de (soi-disant) synonymes comme vieillesse et sénescence, ÉLG 265, Note Sémiologie ; craindre et redouter, CLG/E 330, II B.
} 
mises à sa « sphère de signification » résultent uniquement de la présence ou, respectivement, de l'absence de termes concurrents :

[À propos du mot] autonomie : on se figure qu'il est très important de définir $1^{\circ}$ au sens positif (ce qui est illusoire: qu'on n'épuisera jamais), $2^{\circ}$ au sens immédiat, en quoi consiste l'autonomie d'un peuple pour en tirer $3^{\circ}$ les sens figurés. En réalité, il n'y a pas plutôt un mot d'autonomie que sa sphère de signification est déjà complètement déterminée et uniquement déterminée par l'opposition où il entre avec indépendance, liberté, individualité, etc. de telle façon que, si un seul de ces mots comme indépendance, etc. n'existait pas, aussitôt le sens d'autonomie s'étendrait dans cette direction. (SL n ${ }^{\circ} 105=$ ÉLG 80, Essence double, mes caractères gras)

Si nous reprenons le mot lune, on peut dire, la lune se lève, la lune crô̂t, décroît, la lune se renouvelle, nous sèmerons à la nouvelle lune, il y aura bien des lunes avant que telle chose se produise... et insensiblement nous voyons que $1^{\circ}$ tout ce que nous mettons dans lune est absolument négatif, ne vient pas d'autre chose que de l'absence d'un autre terme, car, et $2^{\circ}$, une multitude d'idiomes exprimeront par des termes tout à fait différents des nôtres les mêmes faits où nous faisons intervenir le mot lune, exprimant par exemple par un premier mot la lune dans ses phases mensuelles, dans un second la lune comme astre différent du soleil, dans un troisième la lune par opposition aux étoiles, dans un quatrième la lune comme flambeau de la nuit, dans un cinquième le clair de lune par opposition à la lune même, etc. Et chacun de ces mots n'a toujours de valeur que par la position négative qu'il occupe par rapport aux autres : ce n'est à aucun moment une idée positive, juste ou fausse, de ce qu'est la lune qui dicte la distribution des notions sous les dix ou douze termes qui existent, mais c'est uniquement la présence même de ces termes qui force de rattacher chaque idée ou au premier ou au second, ou à tous les deux par opposition au troisième et ainsi de suite, sans autre donnée que le choix négatif à faire entre les termes, sans aucune concentration de l'idée diverse sur l'objet un. Ainsi il n'y a jamais rien dans ce mot que ce qui n'était pas d'avance hors de lui ; et ce mot peut contenir et enferme en germe, tout ce qui n'est pas hors de lui. (SL n ${ }^{\circ} 107=$ ÉLG 74-75, Essence double, mes caractères gras)

Pour un signe donné, l'apparition de ce qui semble un nouveau sens ou un nouvel effet de sens est ainsi prévue d'avance, dans un empan que seul l'état des signes contemporains vient délimiter. Intrinsèquement négative, la signification est à la fois sous-spécifiée et indépendante du référent :

On n'empêchera jamais qu'une seule et même chose ne soit appelée selon les cas une maison, une construction, un bâtiment, un édifice, (un monument), un immeuble, une habitation, une résidence, et le contraire serait un signe de notre [ ]. Ainsi l'existence des faits matériels est, aussi bien que l'existence des faits d'un autre ordre, indifférente à la langue. (SL n ${ }^{\circ} 107=$ ÉLG 76, Essence double).

Et du fait que la signification est purement négative, Saussure tire la conclusion qu'il n'y a pas lieu de distinguer entre emploi propre et emploi figuré :

Et ce même fait, purement négatif, de l'opposition avec les mots comparables, est aussi le seul qui fait la justesse des emplois 'figurés'; nous nions en réalité qu'ils soient figurés, parce que nous nions qu'un mot ait une signification positive. Toute espèce d'emploi qui ne tombe pas dans le rayon d'un autre mot n'est pas seulement partie intégrante, mais est aussi 
partie constitutive du sens de ce mot, et ce mot n'a pas en réalité d'autre sens que la somme des sens non réclamés. (SL n ${ }^{\circ} 105=$ ÉLG 80-81, Essence double, mes caractères gras ; voir aussi SL $\left.\mathrm{n}^{\circ} 106-110\right)$

Dans cette perspective, qu'il s'agisse d'un lexème tel que soleil ou d'une marque morphologique, le signifié s'établit de moment en moment en fonction de l'état des signes ambiants. Ipso facto - et en dépit de l'identité des étiquettes qui leur sont appliquées - les catégories telles que 'datif', 'pluriel', 'soleil', etc., changent de contenu d'une langue à l'autre et d'une époque à l'autre ${ }^{39}$. Tels sont, résumés à grands traits, les enseignements d'une application rationnelle de la notion de valeur, qui ouvre sur un questionnement relatif aux universaux et au calcul du sens, et qui mérite d'inspirer non seulement la phonologie, mais aussi la sémantique, la morphosyntaxe, la linguistique contrastive et la typologie des langues.

\subsection{Décrire scientifiquement «ce qui se passe dans la transmission d'une sémiologie ${ }^{40}$}

La continuité qui caractérise la transmission de la langue (cf. supra § 3.1) a pour corrélat le perpétuel «mouvement de la langue dans le temps» (ÉLG 157, Deuxième Conférence à l'Université de Genève) : la langue change, mais sans qu'il y ait jamais rupture avec l'état précédent. Comment rendre compte scientifiquement, c'est-à-dire sans perdre de vue la perspective du locuteur, de ce mouvement si particulier ? Face à cette besogne dont il connaît mieux que personne les difficultés, Saussure s'attache à saisir le changement en $\operatorname{acte}^{41}$ : car tout ce qui est novateur dans la langue y pénètre via l'activité de parole (cf. $\S 2$ et note 15) :

Il faut donc se mettre en face de l'acte de la parole pour comprendre une création analogique. La nouvelle forme je trouve [qui supplante je treuve, MJB] ne se crée pas dans une assemblée de savants discutant sur le dictionnaire. Pour que cette forme pénètre dans la langue, il faut que

$1^{\circ}$ quelqu'un l'ait improvisée, et

$2^{\circ}$ improvisée à l'occasion de la parole, du discours, [...]. (CLG/E 2561-2562, I R, mes caractères gras)

[À propos des préfixes tels que fr. re-] Quelle est la preuve absolue, péremptoire que ces préfixes sont vivants? Ce ne sera que la création analogique, c'est parce que je puis former redémissionner, recontempler, sans les avoir jamais entendues (cf. tous les re- que l'on place devant des mots qui d'après le dictionnaire ne l'admettent pas!). Et cela n'aura lieu qu'à

\footnotetext{
${ }^{39} \mathrm{Cf}$ : : « En revanche ce qui n'existe pas, ce sont

a) les significations, les idées, les catégories grammaticales hors des signes; elles existent peut-être extérieurement au domaine linguistique, c'est une question très douteuse, à examiner en tout cas par d'autres que le linguiste ; ( $\mathrm{SL} \mathrm{n}^{\circ} 32=$ ÉLG 73, Essence double, mes caractères gras). Cf. ibid. 44, 51, 55, 69-70. La question est développée en ces termes dans le Cours III : "Ainsi quand on dit que le grec a gardé le datif indoeuropéen, c'est une simple cö̈ncidence de nom : dans sa forme, le datif grec est très souvent un locatif d'origine, et les fonctions du datif grec ne coïncident que très partiellement avec l'emploi du datif indoeuropéen. C'est une des questions où on risque le plus de prendre des mots pour des choses. Il faut considérer deux points de vue : l'un diachronique, les héritages : l'un synchronique, la répartition des fonctions. Il vaut mieux exprimer la chose par une somme et dire : le latin a gardé six différenciations sur huit cela est très bien : le grec n'en a gardé que quatre. Et non pas : a perdu deux cas ». (CLG/E 1562, II R, mes caractères gras)

${ }^{40}$ ÉLG 262, Note Sémiologie.

${ }^{41}$ Cf. en particulier, sur cette question, Choi 2002, Utaker 2002, Pétroff 2004, Bulea 2006 et 2010.
} 
propos de la parole sans que je réfléchisse, sans que je veuille dire recontempler, etc. ; donc ces préfixes sont bien vivants. (Riedlinger, CLG/E 2590, I R, mes caractères gras)

Ces deux passages du Cours I, dont on rapprochera le passage relatif à je me décolte cité au $\S$ 3.1, suffisent à montrer sans ambiguiité, et à l'inverse de ce qui est enseigné d'habitude, que Saussure accorde un statut primordial à la parole ${ }^{42}$.

La novation une fois ancrée dans l'activité des sujets, il devient possible de distinguer ce qui, dans l'évolution de la langue, relève du changement à proprement parler et ce qui n'en relève pas :

[Suite du passage cité à la note 35] De plus, y a-t-il ici un prétexte quelconque à parler de changement? Aucun; car avant répressionnaire on ne voit point d'équivalent, répressionnaire ne remplace rien, il y a création.

Finals est le pluriel prescrit par le dictionnaire, mais on entend dire aussi finaux. D'autre part, il pourrait arriver qu'un auteur fasse plus tard un adjectif firmamental (de firmament) avec un pluriel firmamentaux. Parlerons-nous alors dans finals-finaux de changement analogique et dans firmamentaux de création analogique ? Nullement, donc dans les deux cas il y a création. Sur le modèle de mur: emmurer on a fait tour : entourer dérivé récent et jour : ajourer (par exemple un travail ajouré). Cela nous apparaît comme une création ; mais si je remarque qu'aux époques précédentes, on disait :

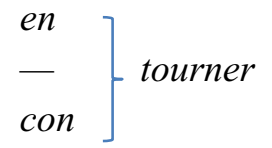

et ajourner (quand tour et jour étaient torn et jorn) alors entourer et ajourer qu'on appelait créations paraissent être plutôt un changement dans nos habitudes par opposition à ajourner et contourner.

$1^{\circ}$ Ces exemples nous montrent la relativité du concept des changements analogiques: nous parlons de changement parce que nous considérons un terme qui a été évincé mais auquel on n'a pas à songer.

$2^{\circ}$ Ils nous montrent que cette conception est incorrecte: les formations que nous qualifions de changements reposent sur le même principe que la création. (CLG/E 2503-2508, II R, mes caractères gras)

Le « changement dans nos habitudes », qui finit par détrôner un terme au profit d'un autre, ne doit pas, explique Saussure, masquer le fait que l'analogie consiste en une création à partir de modèles, dans laquelle la forme supplantée ne joue aucun rôle; même si les formes analogiques participent à la transformation de la langue, elles ne sont pas en elles-mêmes des transformations ${ }^{43}$. Ne relève pas non plus de la transformation ce qui est appelé -

\footnotetext{
${ }^{42}$ Cf. $\S 2$ et note 15 . La priorité donnée à la linguistique de la langue dans le CLG a été orchestrée par les éditeurs, comme le montre une confrontation des passages concernés à leurs sources (Béguelin 2011). Saussure écrit quant à lui : «La linguistique, j'ose le dire, est vaste. Notamment elle comporte deux parties : l'une qui est plus près de la langue, dépôt passif, et l'autre qui est plus près de la parole, force active et origine véritable des phénomènes qui s'aperçoivent ensuite peu à peu dans l'autre moitié du langage. " (ÉLG 273, Rapport sur la création d'une chaire de stylistique). Voir aussi Bouquet 1997 et 2010, Rastier 2003, Kyheng 2005, Laks 2012.

${ }^{43}$ «Au point de vue général de la langue, les créations analogiques entraînent en effet un changement : quelque chose est créé et quelque chose est abandonné. Dans ce cas, quand on parle de l'évolution de la langue, on peut
} 
abusivement selon lui - le changement de signification, qui n'est autre que le résultat de l'application du principe de négativité dont relèvent les signifiés (cf. § 3.2). Le changement phonétique, au contraire, procède par substitution d'un terme à un autre et met en jeu deux époques $^{44}$ :

On est plongé dans une profonde rêverie en voyant comparer, dans des ouvrages sérieux (Exemple Whitney [...]), ces deux espèces de changement dans le temps :

a) un mot change de signification ;

b) un mot change de forme (ou de son), enfin change matériellement.

Il faudrait tout reprendre, et on ne sait par quel côté commencer. Il faudrait, entre mille choses, demander ce qu'est un mot (dans le temps), s'il peut changer de forme et de signification, et dès lors ce que signifie l'affirmation seule qu[ ]

Mais bornons-nous à ressaisir le fil directeur au lieu d'essayer de débrouiller l'entassement d'erreurs et de termes mal définis qui [ ]

Nous poserons alors en entrant dans le cadre inadmissible :

- que le changement de signification n'a point de valeur comme fait résultant du temps, pour toutes sortes de raisons, entre autres que ce changement est de tous les instants et n'exclut pas la signification précédente qui devient concurrente ; tandis que le changement de forme réside dans la substitution d'un terme à un autre; et que cette substitution consacre, suppose avec nécessité, et suppose seule la présence successive de deux époques ;

- que la signification n'est qu'une façon d'exprimer la valeur d'une forme, laquelle valeur dépend complètement des formes coexistantes à chaque moment, et que c'est par conséquent une entreprise chimérique, non-seulement de vouloir suivre cette signification en elle-même (ce qui n'est plus du tout linguistique), mais même de vouloir la suivre par rapport à une forme, puisque cette forme change, et avec elle toutes les autres, et avec celles-ci toutes les significations de manière qu'on ne peut dominer le changement de signification que vaguement par rapport à l'ensemble mais non [ . (SL no $128=$ ÉLG 41, Double Essence, mes caractères gras)

La plasticité ou dilatabilité intrinsèque de la signification (De Palo 2015 : 120 et passim), les accidents qui affectent, à tout instant, l'ensemble du système linguistique, le fait que la valeur du signe ne procède que de ses rapports avec ses contemporains et que, contrairement aux apparences, cette valeur ne se transmet pas dans le temps : autant de facteurs qui rendent illusoire aux yeux de Saussure la prétention de procéder, sans tomber dans l'approximation, à une histoire sémantique des formes ${ }^{45}$.

dire que le phénomène analogique est une force transformatrice de la langue, bien que les créations analogiques ne soient pas des transformations. » (CLG/E 2570, I R)

${ }^{44}$ D'où l'identification que fait Saussure entre instantané et morphologique d'une part, successif et phonétique de l'autre : «Le fait qu'il n'y a rien d'instantané qui ne soit morphologique (ou significatif); et qu'il n'y a rien non plus de morphologique qui ne soit instantané est inépuisable dans les développements qu'il comporte.

Mais ce premier fait a pour contre-partie immédiate :

qu'il n'y a rien de successif qui ne soit phonétique (ou hors de la signification), et qu'il n'y a rien de phonétique qui ne soit successif. » (SL n ${ }^{\circ} 112$ = ÉLG 41, Double Essence ; cf. aussi 67-70)

${ }^{45}$ Cf. note précédente et ÉLG 40-43, notamment : «La persistance (plus ou moins exacte) de beaucoup de fonctions significatives dans le temps et dans les formes est le fait qui nous suggère faussement l'idée - je ne dis pas qu'il existe une histoire des significations, parce que cela ne signifie décidément rien -, mais qu'il existe une histoire de la langue prise par le double côté de la forme et du sens (c'est-à-dire une morphologie historique $):[\ldots] »\left(\mathrm{SL} \mathrm{n}^{\circ} 12=\right.$ ÉLG 41, Essence double $)$. 
Le paradoxe qui saute aux yeux de Saussure, et auquel il se heurte lui-même dans sa tâche quotidienne de professeur de grammaire comparée et d'historien des langues, c'est que les entités considérées à travers le temps échappent à la sémiologie : extraites des systèmes où réside leur valeur, elles ne sont que des enveloppes vocales privées de statut linguistique ${ }^{46}$. Le concept d'identité diachronique, du fait qu'il est «sans corrélatif dans les faits » est donc éminemment suspect et doit être soumis à critique ${ }^{47}$ :

Il est mystérieux, le lien de cette identité diachronique, qui fait que deux mots ont changé complètement (calidus : šo; aiwa:je) et qu'on en affirme cependant l'identité. En quoi consiste-t-il ? Précisément ! Il y aura donc dans la linguistique toute une série de questions à résoudre, ou plutôt à scruter qui se rapportent aux identités, unités diachroniques. (CLG/E I, $2742-3$ et 2740 , II R) $)^{48}$

Une fois reconnues la nature anti-historique du langage (ÉLG 216, Notes pour un article sur Whitney) et l'inexistence d'une "grammaire historique » ${ }^{49}$, est-il encore possible de décrire scientifiquement le changement morphologique? La note Morphologie apporte une réponse en ces termes :

Le changement morphologique nécessite une étude spéciale qui prend le nom de Morphologie historique. Elle sépare les époques et les compare, tandis que la morphologie rétrospective les confond. Elle nous présente la véritable perspective entre les classifications et les interprétations successives auxquelles la langue a pu se livrer sur les mêmes formes, tandis que la morphologie rétrospective cherche, si vous me permettez cette image, à obtenir la projection sur un même plan de classifications très différentes par leur date. (ÉLG 188, Note Morphologie, mes caractères gras ${ }^{50}$ ).

\footnotetext{
${ }^{46}$ Et qui sont même, de ce fait, qualifiées de « cadavres » dans les Anciens Item : ÉLG 107.

${ }^{47} \mathrm{Cf}$. la citation du début du $\S 3$ 3.2. et aussi : «L'identité étymologique (notion purement grammaticale, qui n’a aucun corrélatif dans les faits, [...]) est celle par laquelle nous imposons idéalement à un état de langue $\mathrm{B}$ une identité morphologique de l'état $\mathrm{A}$ appartenant au passé - qui s'est vue brisée ou effacée par une cause quelconque. » (SL n ${ }^{\circ} 132$, qui corrige le passage correspondant de ÉLG 84-85, Essence double; le terme de grammatical est à prendre ici au sens de 'savant', 'artificiel').

${ }^{48}$ Voici la version 'politiquement correcte' de ce passage proposée par Bally \& Sechehaye : «Or l'identité diachronique de deux mots aussi différents que calidum et chaud signifie simplement que l'on a passé de l'un à l'autre à travers une série d'identités synchroniques dans la parole, sans que jamais le lien qui les unit ait été rompu par les transformations phonétiques successives. (CLG 250, mes caractères gras; l'emploi de l'adverbe simplement est pour le moins déplacé en l'occurrence ; cf. ÉLG 200-201, Notes pour un livre sur la linguistique générale 2).

49 «Il n'y a pas pour nous de grammaire historique : les termes jurent ensemble; il n'y a pas de système qui puisse être à cheval sur une suite d'époques. Ce qui est synchroniquement dans une langue est un équilibre qui se réalise de moment en moment. » (CLG/E 2129, II R).

${ }^{50}$ Une critique détaillée de la dite «morphologie rétrospective» est fournie dans la même note : "Autre exemple : en français de nos jours enfant, entier ne comportent au sentiment des Français aucune espèce d'analyse, pas plus que n'en comporterait le mot pour ou le mot moi.

Au premier siècle, infans, integer, qui correspondent phonétiquement, comportent une analyse, car par exemple in-auditus et färi, tango, etc. permettent à la langue de décomposer ainsi : in-fans, in-teger.

Si je me mets à couper en-fant, en-tier, je fais la même chose que tout à l'heure : de la morphologie latine sur des formes françaises.

Eh bien, c'est cette morphologie-là qui est la base de toutes les grammaires gréco-latines. C'est cette morphologie-là que nous allons faire nous aussi, dans la moitié des cas. Seulement, vous aurez été dûment avertis et mis en état, je l'espère, de vous rendre compte de sa véritable valeur. » (ÉLG 186, Note Morphologie)
} 
La démarche préconisée pour l'étude du changement grammatical se concrétise dans la «figure simple» du carré linguistique, outil à la fois méthodologique et didactique omniprésent dans l'œuvre de Saussure. Dans ce carré, les axes horizontaux - seuls nécessaires! - reflètent le point de vue subjectif des locuteurs d'une époque donnée ; les axes verticaux représentent quant à eux un point de vue "objectif», purement savant et artificiel, car « sans corrélatif dans les faits » $\left(\mathrm{SL} \mathrm{n}^{\mathrm{0}} 132=\right.$ ÉLG 84) :

\section{Carré linguistique.}

Toutes considérations possibles sur un fait linguistique sont immédiatement enfermées en une figure simple et partout la même, comprenant quatre termes :

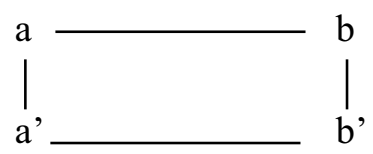

Le sens vertical marquant la valeur du temps, et la distance horizontale [ ].

Les rapports possibles sont marqués par les lignes. Il n'y a aucun rapport possible par exemple entre $\mathrm{a}$ et b' ou b et a', selon une ligne diagonale.

À une condition, savoir qu'on ne veut appliquer aucun esprit historique aux faits, la figure non seulement pourra, mais devra se réduire à $\mathbf{a}-\mathbf{b}$.

Nous n'avons que quatre principes :

Le premier qu'il faut distinguer l'événement de l'état.

Le second qu'il faut les opposer, par exemple subordonner l'un à l'autre.

Le troisième qu'il faut les séparer; cela en outre d'une manière tellement absolue que la seule question qui reste est de savoir [ ]

Le quatrième enfin que toute autre façon de classifier est vaine jusqu'à un degré dont rien ne peut donner l'idée. (ÉLG 228-229, Status et motus. Notes pour un livre de linguistique générale, 1 , mes caractères gras)

Par confrontation de deux états successifs, le carré linguistique restitue la perspective des communautés parlantes concernées, en même temps qu'elle met en lumière la rupture du lien grammatical favorisée par l'évolution phonétique :

Un mot et ce qui dans la conscience de tout le monde est son dérivé, deviennent, chacun étant abandonné aux vicissitudes phonétiques, des mots séparés.

$$
\begin{array}{cc} 
& \text { (vervex } \sim \text { vervecarius) } \\
\text { lat. pop. } & \text { berbix } \sim \text { berbicarius } \\
& \downarrow \\
& \text { brebis } / / \text { berger: }
\end{array}
$$

dans le parler local a même le sens de gardien de bøufs !

On ne peut pas parler de rapports étymologiques entre brebis et berger. L'étymologie n'a aucune place dans la linguistique. L'adjectif étymologique est un mot qui a sa clarté et qui résume toute espèce de phénomènes linguistiques. Mais comme nous voulons expliquer ce qui existe dans le phénomène, nous ne dirons pas que la rupture du lien grammatical est l'effet d'un fait étymologique mais d'un changement phonétique. (CLG/E 2360-2364, I R ; sont traités dans cette même partie les exemples de onze, ferté, ennemi, enseigner, all. Drittel etc.)

Comme le montre cet extrait, la matière par excellence de la morphologie historique réside dans les réinterprétations auxquelles les sujets parlants soumettent les faits langagiers, 
réinterprétations qui traduisent une modification des rapports entre les formes; le changement grammatical est ainsi ramené à un pur et simple déplacement des valeurs. Dans le domaine syntaxique, ce déplacement peut intervenir à la faveur d'une agglutination (tous jours $>$ toujours, lieu tenant > lieutenant, choisir ai > choisirai), phénomène « passif » qui reflète une tendance à la « simplification de l'idée » et " puise son existence dans la sphère de la phrase » (CLG/E 2684 et 2709, I R) ; il peut également résulter de «quiproquos », considérés par Saussure comme des « agents réguliers de la transformation du langage » :

Une masse énorme d'innovations se résume ainsi en véritables quiproquos ; le quiproquo est [ ] doit être reconnu comme un des agents réguliers de la transformation du langage. Exemple du quiproquo typique: on dit aujourd'hui une nouvelle à la main, parce que dans le titre Nouvelles à la main (comme l'épée au côté, sac sur le dos) le rapport véritable entre à la main et nouvelles a été mésinterprété. (Note datée de mars 1885, in Marchese 2015, 186, mes caractères $\operatorname{gras}^{51}$ )

Quoi qu'il en ait: D'où vient cette locution ? C'est qu'on dit malgré (mal gré) que j'en aie. Il y a eu une légère altération de malgré, et on l'a identifié avec quoique. (CLG/E 336, B)

Autre exemple : de par le roi = pour la part du roi (par pour part, le roi servait de génitif); exemple tout à fait simple : Quand deux mots arrivent à n'en faire plus qu'un, ce qui amène un quiproquo et une altération de sens. (CLG/E 336, II R, mes caractères gras)

La continuité dans la transmission de la langue est donc faite de discontinuités successives, occasionnées par les décompositions et recompositions auxquelles l'esprit des locuteurs soumet le donné linguistique ${ }^{52}$ : ainsi, l'emploi de nouvelle à la main au sens d' 'anecdote' 'histoire drôle' suppose une réinterprétation démotivante de la locution nouvelles à la main, longtemps utilisée au sens de 'gazettes ou pamphlets manuscrits' (et qui en 1843 a donné son titre à un vaudeville ; cf., dans le même esprit, l'apparition du singulier fait divers, qui résulte d'une démotivation de l'adjectif dans la locution plurielle faits divers). Quant au sort réservé à mal gré que j'en aie, de part le roi, il illustre les réanalyses imprévisibles entraînées par l'existence, à côté de la séquence concernée, de telle ou telle forme collatérale (parasynonyme quoique dans le premier cas, préposition par homophone du substantif part dans le second).

La mutabilité de la langue, aux yeux de Saussure, est ainsi marquée du sceau de la fortuité : c'est le plus parfait hasard, sous la forme de tel ou tel changement phonétique, ou sous la forme de telle ou telle circonstance paradigmatique aléatoire, qui préside à l'installation ou à la rupture du lien grammatical :

Par sa genèse, un procédé provient de n'importe quel hasard. Par exemple, Hôtel-Dieu (signifiant exactement au moyen âge Hôtel de Dieu) offre un procédé totalement identique à l’hébreu [tsédek Yahweh] 'justice de Dieu'. Le procédé 'hébreu' est employé sans restriction dans des centaines de juxtaposés français.

\footnotetext{
${ }^{51}$ Je n'ai pas reproduit ici les passages biffés du manuscrit. Cf. un peu plus haut : « on sait le sens de la phrase mais on analyse mal les rapports qui existent entre ses différents termes ». (Ibid.)

${ }^{52}$ On parlerait aujourd'hui, plutôt que de quiproquo, de métanalyse ou de réanalyse (Béguelin 2010, 2014, Béguelin et al. 2015).
} 
[ ... ] le tout se passe hors de l'esprit, dans la sphère des mutations de sons, qui bientôt imposent un joug absolu à l'esprit, et le forcent d'entrer dans la voie spéciale qui lui est laissée par l'état matériel des signes.

[...] Le 'génie de la langue' pèse zéro en face d'un seul fait comme la suppression d'un $o$ final, qui est à chaque instant capable de révolutionner de fond en comble le rapport du signe et de l'idée, dans n'importe quelle forme de langage, précédemment donnée [ ]; (ÉLG215-216, Notes pour un article sur Whitney)

Cette conception de l'évolution linguistique est anti-schleichérienne et résolument non déterministe : le changement n'obéit à aucune « voie fatale » (ibid.), il n'est pas calculable par le logicien ${ }^{53}$ — bien que Saussure admette l'existence d'une tendance à la « simplification de l'idée » qui préside aux agglutinations.

La position de Saussure, une fois de plus, est singulière : par une application intransigeante de la théorie de la valeur, par le refus d'envisager la destinée d'une forme hors de celles de ses collatérales, elle rompt avec des préjugés profondément ancrés, qui orientent aujourd'hui encore les approches dominantes du changement linguistique (foi accordée à la notion d'identité diachronique, idée d'une gradualité et d'une directionnalité des changements).

\subsection{La raison du locuteur}

Quels que soient le fait linguistique et l'époque considérés, la méthode saussurienne revient on l'a vu à calquer le point de vue du locuteur. Pas de demi-mesure à cet égard : l'empathie doit aller jusqu'à épouser les «fausses » analyses du sujet parlant. Dans une étude de 1905 intitulée «D' $\Omega$ MH $\Lambda$ Y $\Sigma$ I $\Sigma$ à TPIחTO $\Lambda$ EMO $\Sigma »$, Saussure réfute l'étymologie en usage pour le

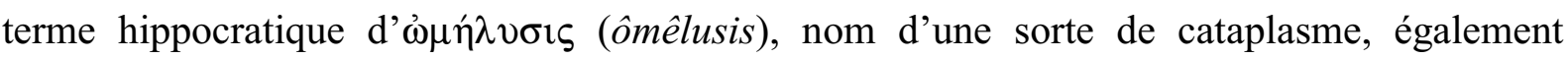

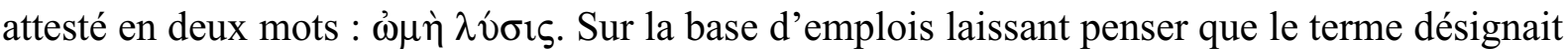

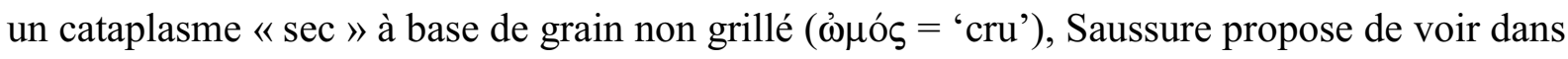

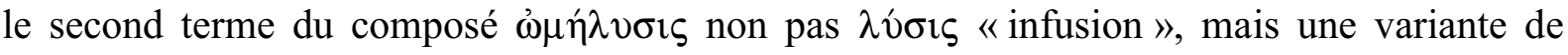

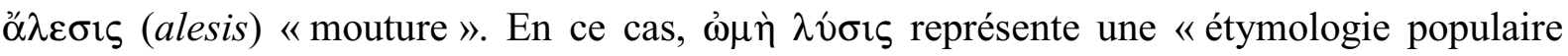
imparfaite, ou 'inachevée'», dans laquelle un des termes demeure "privé de sens, livré à l'inconnu, sans essai d'interprétation ». Saussure appuie cette hypothèse par un exemple tiré du lexique médical français :

En choisissant parmi une collection de termes populaires français que j'ai sous les yeux par hasard, un cas pouvant particulièrement fournir le pendant du fait, je n'ai pas de peine à le trouver dans : de l'eau d'anum, qui signifie, parait-il, du laudanum, mais où on voit comment

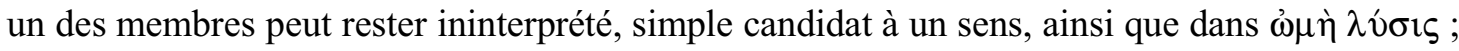
les deux mots [i. e. le mot grec et le mot français, MJB] relevant d'ailleurs de la langue médicale, placée au premier chef dans la sphère du lexique où ces déformations se produisent d'habitude. (Recueil 578)

Comme bien d'autres passages de l'œuvre de Saussure, cette recherche démontre que face à ses contradicteurs, le linguiste ne peut avoir raison qu'en épousant au plus près la raison du locuteur.

\footnotetext{
${ }^{53}$ Cf. De Mauro, CLG/DM n. 176 p. 454 ; Béguelin 2010.
} 
C'est une entreprise qui peut être en général taxée, en étymologie, de jeu très pitoyable que de s'attaquer à des formes limpides pour vouloir y trouver autre chose que ce qu'elles donnent d'emblée. Mais les entreprises de l'étymologie populaire égalent ou dépassent en ingéniosité celles du grammairien, c'est là le facteur à ne jamais oublier complètement. (Recueil 582, mes caractères gras)

Dans ses leçons de linguistique générale (Cours I), Saussure fournit une systématisation remarquable des phénomènes d'étymologie populaire, dont Bally et Sechehaye — sous l'effet de leurs réticences personnelles à l'égard des calembours - ne donnent qu'une version banalisée et censurée (Béguelin 1995). Saussure apportait, selon son habitude, abondance d'exemples dont les plus originaux sont absents du CLG : ainsi langue verte, utilisé pour Landwehr dans certains départements français pendant la guerre franco-allemande; huile de foie d'amoureux pour huile de foie de morue (CLG/E 2655: I R); trois cadéros pour Trocadéro (avec pour effet de laisser, comme dans le cas de l'eau d'anum un "reste " « candidat à un sens ») :

Trocadéro à Paris est appelé dans une partie de la population parisienne trois cadéros, (je vais aux trois cadéros), peut-être à cause de son architecture particulière (deux tours mauresques et $<$ une partie> centrale qui a aussi la forme de $\left.\operatorname{tour}^{54}\right)$. (CLG/E 2658 : I R)

Saussure disqualifie, on l'a vu plus haut, l'étymologie savante ; en contrepartie, il réhabilite l'étymologie populaire qui, si dépréciée soit-elle, donne accès aux représentations linguistiques du locuteur, à son savoir épilinguistique. Ce que Bally et Sechehaye n'ont pas compris (ou n'ont pas voulu comprendre), c'est que seules sont réellement pathologiques aux yeux de Saussure les analyses qui envisagent la langue d'un point de vue que le locuteur ne saurait partager.

\section{Conclusions}

Le comportement linguistique, les compromis successifs qui s'établissent entre les signes et l'esprit des locuteurs, tels sont les objets fondamentaux de la linguistique saussurienne ; et la fusion revendiquée entre perspective du linguiste et perspective du locuteur impose, comme le montrent les exemples traités ci-dessus, de décrire tous les faits langagiers, quel que soit leur statut au regard des normes en vigueur. Dans son activité empirique, Saussure recourt constamment à la langue vivante : les postulats qui gouvernent sa théorie — primauté du point de vue du locuteur, identité négative des signes - sont régulièrement confrontés aux données d'observation, et de manière symétrique, le moindre exemple est traité à l'aune des grands principes. Clairvoyant et pessimiste à la fois, Saussure pointe un idéal descriptif qu'il juge luimême inaccessible ${ }^{55}$; son travail empirique n'en illustre pas moins, au jour le jour, l'opérationnalité de sa théorie.

\footnotetext{
${ }^{54}$ Le monument auquel il est fait référence ici est celui qui a précédé l'actuel Palais de Chaillot.

${ }^{55}$ «Faut-il dire notre pensée intime ? Il est à craindre que la vue exacte de ce qu'est la langue ne conduise à douter de l'avenir de la linguistique. Il y a disproportion, pour cette science, entre la somme d'opérations nécessaires pour saisir rationnellement l'objet, et l'importance de l'objet : [...] » (SL n ${ }^{\circ} 24=$ ÉLG 87, Essence double).
} 
Le Professeur genevois a été immergé sa vie durant dans la linguistique diachronique, domaine où il mérite tout particulièrement d'être entendu. La méthode historique qu'il préconise passe, on l'a vu, par la description d'états de langues successifs, avec en ligne de mire le système dans sa globalité ; le changement morphosyntaxique ne saurait être imputé, dans sa perspective, à une quelconque vocation interne à l'entité considérée, car l'entité est dépourvue de substrat positif, et le changement prend naissance dans l'activité du locuteur, sous la pression de paramètres extérieurs à cette entité. À l'évidence, il y a ici de quoi stimuler la réflexion des jeunes linguistes qui abordent les études diachroniques en se fiant ingénument à la notion d'échelle ou de parcours de grammaticalisation (cf. Béguelin 2010).

Pour conclure au moins provisoirement, retenons que la position de Saussure n'est pas immanentiste au sens caricatural où elle a été longtemps dépeinte. Contrairement à ce qui en est dit dans le CLG, Saussure conçoit le couple langue-parole comme une dualité, non comme une dichotomie ; il légitime explicitement l'existence d'une linguistique de la parole (cf. note 42). Comme l'a relevé son élève Regard (note 29), Saussure est également sensible aux facteurs externes qui agissent sur le devenir des langues : l'explication qu'il propose dans le cas de trois cadéros en fournit une preuve parmi d'autres. Et comme l'indiquent les notes prises par les auditeurs du Cours III, la priorité donnée à la description du système n'a pour Saussure rien d'exclusif: elle vise à doter la linguistique d'un point de départ, en vue d'aborder sur des bases solides les diverses dimensions du langage ${ }^{56}$.

\section{Références}

\section{Textes issus du corpus saussurien}

CLG : Cours de linguistique générale, $1^{\mathrm{e}}$ éd. par Bally, C. \& A. Sechehaye, $1916 ; 2^{\mathrm{e}}$ éd. 1922 ; $3^{\mathrm{e}}$ éd. 1931 ; CLG/DM : éd. critique par De Mauro, T. Paris : Payot, 1972.

CLG/E : Cours de linguistique générale, édition critique par Engler, R., tome I (1968), tome II, appendice (1974). Wiesbaden : Harrassowitz.

Cours III C : Constantin, E. (2005). Linguistique générale. Cours de M. le professeur F. de Saussure. Gambarara, D. \& C. Mejía Quijano, éds. Cahiers Ferdinand de Saussure 58, 71-289.

ĖLG : Écrits de linguistique générale (2002). Bouquet, S. \& R. Engler (éds) Paris : Gallimard.

Légendes: «La légende de Sigfrid et l'histoire burgonde », «Le cycle de Dietrich », «Tristan », «Précisions théoriques ». Turpin, B. (Ed.) (2003). Cahiers de l'Herne Saussure 76, 351-429.

Leçons: Saussure, F. de, en préparation. Leçons de linguistique générale. Bouquet, S. \& A. Weil, éds.

\footnotetext{
${ }^{56}$ «On peut en outre dire que c'est en choisissant la langue comme centre et point de départ qu'on a la meilleure plateforme pour aller aux autres éléments du langage. » (CLG/E 321 et 323, III C).
} 
Marchese, M. P., éd. (2015). Deux manuscrits de Saussure datés 1884 et 1885 (ADS 374/1 P. 315-320. CFS 68, 179-187.

Mejía Quijano, C., éd. (2014). Ferdinand de Saussure. Une vie en lettres. 1866-1913. Nantes, Éditions nouvelles Cécile Defaut.

Mémoire: Mémoire sur le système primitif des voyelles dans les langues indoeuropéennes (1879). In Recueil des publications scientifiques de Ferdinand de Saussure (1922) (pp. 1-268). Bally, C. \& L. Gautier, éds. Genève : Sonor [réimpression Slatkine, 1984].

Phonétique: Phonétique. Il manoscritto di Harvard Houghton Library bMS Fr 266 (8). Edizione a cura di Marchese, M. P. (1995). Padova : Unipress.

Recueil: Recueil des publications scientifiques de Ferdinand de Saussure (1922). Bally, C. \& L. Gautier, éds. Genève : Sonor [réimpression Slatkine, 1984].

Saussure, F. de (2013). Anagrammes homériques, Limoges : Lambert-Lucas

SL : Saussure, F. de (2011), Science du langage. De la double essence du langage et autres documents du ms. BGE Arch. de Saussure 372, éd. critique partielle mais raisonnée et augmentée des Écrits de linguistique générale établie par R. Amacker. Genève : Droz.

Sofia, E. (2016). La «Collation Sechehaye» $d u$ 'Cours de linguistique générale' de Ferdinand de Saussure. Introduction, transcription et notes par Estanislao Sofia. Louvain : Peeters.

Théorie des sonantes: Théorie des sonantes. Il manoscritto di Ginevra BPU Ms. fr. 3955/1. Edizione a cura di Marchese, M. P. (2002). Padova : Unipress.

\section{Autres références}

Arrivé, M. (2009). La rime dans l'enseignement de Ferdinand de Saussure. Cahiers Ferdinand de Saussure 62, 103-115.

Béguelin, M.-J. (2003). La méthode comparative et l'enseignement du Mémoire. Cahiers de l'Herne - Saussure, 76, 150-164.

Béguelin, M.-J. (2010). Le statut des 'identités diachroniques' dans la théorie saussurienne : une critique anticipée du concept de grammaticalisation. In Bronckart, J.P., Bulea, E. \& Bota, C., éds, 239-269.

Béguelin, M.-J. (2011a). Langue reconstruite et langue tout court. Cahiers Ferdinand de Saussure 62, 9-32.

Béguelin, M.-J. (2011b). Linguistique de la langue et linguistique de la parole. In : G. Corminboeuf \& M.-J. Béguelin, éds. Du système linguistique aux actions langagières. Mélanges en l'honneur d'Alain Berrendonner. Bruxelles : De Boeck-Duculot, 641-661. 
Béguelin, M.-J. (2012). La place de la grammaire comparée. Langages 185, 75-90.

Béguelin, M.-J. (2014). Deux points de vue sur le changement linguistique. Langages 196, 13-36.

Béguelin, M.-J. (à par. 2016). Le changement morphologique selon Saussure. Volume d'hommages.

Béguelin, M.-J., Corminboeuf, G. \& Johnsen, L. A. (2015). Réflexions sur le statut de la réanalyse. Verbum tome XXXVI, no 1, 3-16.

[Voir aussi sous : [Reichler-] Béguelin, M.-J.]

Benveniste, E. (1963). Saussure après un demi-siècle. Cahiers Ferdinand de Saussure 20, 7-21 (repris dans Problèmes de linguistique générale I (1966), Paris : Gallimard, 32-45).

Bergounioux, G. (2010). La phonologie comme morphologie. In Bronckart, J.-P., E. Bulea \& C. Bota, éds, 105-124.

Bergounioux, G. \& B. Laks (2003). Portrait de Saussure en phonologue contemporain. In Bouquet, S. éd. Saussure. Paris : Cahiers de L'Herne, 165-178.

Bouquet, S. (1997). Introduction à la lecture de Saussure. Paris : Payot.

Bouquet, S., éd. (2003). Saussure. Paris : Cahiers de L’Herne.

Bouquet, S. (2010). Du pseudo-Saussure aux textes saussuriens originaux. In Bronckart, J.-P., Bulea, E. \& Bota, C., éds, 31-48.

Bronckart, J.-P., E. Bulea \& C. Bota, éds (2010). Le projet de Ferdinand de Saussure. Genève : Droz.

Bulea, E. (2006). La nature dynamique des faits langagiers, ou de la «vie» chez Ferdinand de Saussure. Cahiers Ferdinand de Saussure 59, 5-20.

Bulea, E. (2010). Le défi épistémologique de la dynamique temporalisée. In Bronckart, J.P., Bulea, E. \& Bota, C., éds, 215-238.

Choi, Y. H. (2002). Le problème du temps chez Ferdinand de Saussure. Paris: L'Harmattan.

De Palo, M. (2016). L'invention de la sémantique. Bréal et Saussure. Traduit de l'italien par A. M. Perrone. Préface de F. Gandon. Limoges : Lambert-Lucas.

Depecker, L. (2009). Comprendre Saussure. Paris : Armand Colin.

Depecker, L. (éd.) (2012). Les manuscrits de Saussure. Une révolution épistémologique. Langages 185. 
Coursil, J. (2015). Valeurs pures. Le paradigme sémiotique de Ferdinand de Saussure. Limoges : Lambert-Lucas.

Fehr, J. (2000). Saussure entre linguistique et sémiologie. Paris : PUF.

Forel, C., E. Paillard \& P.-Y. Testenoire (2015). Saussure et l'Antiquité aujourd'hui. Une introduction. Cahiers Ferdinand de Saussure 68, 89-93.

Gadet, F. (1987). Saussure. Une science de la langue. Paris : PUF.

Godel, R. (1969 = 1957). Les sources manuscrites du Cours de linguistique générale de F. de Saussure. Genève : Droz ( $2^{\mathrm{e}}$ tirage, 1969).

Joseph, J. (2012). Saussure. Oxford, Oxford University Press.

Kuryłowicz, J. (1978). Lecture du Mémoire en 1978 : un commentaire. Cahiers Ferdinand de Saussure 32, 7-26.

Kyheng, R. (2005).Langue et parole : dichotomie ou dualité ? Texto ![en ligne], URL : http://www.revue-texto.net/Saussure/SurSaussure/Kyheng/Kyheng langue.html (consulté le 20 octobre 2011).

Kyheng, R. (2007), Principes méthodologiques de constitution et d'exploitation du corpus saussurien, Texto![en ligne], URL : http://www.revue-texto.net/index.php?id=1796. (consulté le 5 juin 2011).

La Fauci, N. (2005). Principes de linguistique rationnelle, Pisa : Edizioni ETS.

La Fauci, N. (2011). Relazioni e differenze. Questioni di linguistica razionale, Palermo: Sellerio.

Laks, B. (2012). La phonotactique saussurienne : système et loi de la valeur. Langages $185,65-73$.

Pétroff, A.-J. (2004). Saussure : la langue, l'ordre et le désordre. Paris : L’Harmattan.

Rastier, F. (2003). Le silence de Saussure ou l'ontologie refusée. Cahiers de l'HerneSaussure, 76, 23-51.

Rastier, F. (2012). Lire les textes de Saussure. In : Depecker, L., éd., 7-20.

Rastier, F. (2015). Saussure au futur. Paris : Les Belles Lettres, Encre Marine.

Redard, G. (1978). Deux Saussures ? Cahiers Ferdinand de Saussure 32, 27-41.

[Reichler-]Béguelin, M.-J. (1990a). Des formes observées aux formes sous-jacentes. In Amacker, R. \& R. Engler, éds. Présence de Saussure. Genève : Droz, 21-37.

[Reichler-]Béguelin, M.-J. (1990b). Conscience du sujet parlant et savoir du linguiste. In Liver, R., I. Werlen \& P. Wunderli, éds. Sprachtheorie und Theorie der 
Sprachwissenschaft. Festschrift für Rudolf Engler. Tübingen: Gunter Narr Verlag, 208220.

[Reichler-]Béguelin, M.-J. (1995). Saussure et l'étymologie populaire. In Normand, C. \& M. Arrivé, éds. Saussure aujourd'hui, Actes du Colloque de Cerisy (12-19.8.1992). Numéro spécial de $\operatorname{LINX}$ (pp. 121-138). Paris : Université Paris X-Nanterre.

[Reichler-]Béguelin, M.-J. (2000). Des coefficients sonantiques à la théorie des laryngales. In Auroux, S. éd. Histoire des idées linguistiques, tome III, Bruxelles: Mardaga, 173-182.

Rousseau, A. (2006). Ferdinand de Saussure descripteur des langues - À la lumière d'un cours inédit sur le gotique (1890-1891). In de Saussure, L. éd., 71-94.

Saussure, L. de (éd.) (2006). Nouveaux regards sur Saussure. Mélanges offerts à René Amacker. Genève : Droz.

Starobinski, J. (1971). Les mots sous les mots. Les anagrammes de Ferdinand de Saussure. Paris : Gallimard ; rééd. 2009, Limoges : Lambert-Lucas.

Testenoire, P.-Y. (2013). Ferdinand de Saussure à la recherche des anagrammes, Limoges, Lambert-Lucas.

Utaker, A. (2002). La philosophie du langage. Une archéologie saussurienne. Paris: PUF ; rééd. 2016, Limoges : Lambert-Lucas.

Vallini, C. (2013). Studi saussuriani. Naples: Università degli studi di Napoli, « L'Orientale».

Watkins, C. (1975). Remarques sur la méthode de Ferdinand de Saussure comparatiste. Cahiers Ferdinand de Saussure 32, 59-69. 